\title{
Thoracoscopic approach to the resection of an azygos vein aneurysm
}

Thomas D. Person, MD, Christopher B. Komanapalli, MD, Hannan Chaugle, MD, Paul H. Schipper, MD, and Mithran S. Sukumar, MD, Portland, Ore

I diopathic azygos vein aneurysms are uncommon. Approximately 30 cases have been reported in the literature to date. ${ }^{1}$ In many of these cases, the aneurysm was asymptomatic. However, reported symptoms have included mass effect with superior vena caval compression, right main stem bronchus compression, and pulmonary embolism. This case is the first report of presyncopal symptoms as a result of an azygos vein aneurysm.

\section{Clinical Summary}

A 61-year-old woman presented to the emergency department with lightheadedness, nausea, and leg weakness. Her past medical history was significant for hypothyroidism, for which she was taking levothyroxine (Synthroid). Her initial evaluation included orthostatic vital signs, which revealed significant orthostatic hypotension. Her chest radiograph was grossly normal, and a computed tomographic scan of the chest revealed a posterior mediastinal mass (Figure 1) that was enhancing. This was believed to be consistent with a mediastinal tumor, and magnetic resonance imaging of her chest and spine confirmed this mass without yielding another source for her symptoms. She underwent endoscopic ultrasonography through the esophagus that revealed an anechoic mass with weak Doppler signals. Needle aspiration yielded only blood elements. She was referred to our institution and, after evaluation by means of pulmonology and thoracic surgery, was approved for thoracoscopic resection of her mediastinal mass. After appropriate patient positioning, 3 thoracoscopic ports were placed. The lung was deflated to reveal an aneurysmal dilation of the azygos vein (Figure 2). The vagus nerve was noted to be running in close proximity and might have been responsible for the patient's symptoms. Proximal and distal control was obtained after incision of the pleura and encircling the azygos vein. Then a linear vascular stapling device (US Surgical, Norwalk, Conn) was used to resect the aneurysm. After removal of the aneurysm, the incisions were closed, and the patient was taken to the intensive care unit for observation overnight. She was then transferred to the

From the Division of Cardiothoracic Surgery, Oregon Health \& Sciences University, Portland, Ore.

Received for publication Nov 5, 2004; accepted for publication Nov 24, 2004.

Address for reprints: Mithran S. Sukumar, MD, Oregon Health \& Sciences University, Department of Surgery, Division of Cardiothoracic Surgery L353, 3181 SW Sam Jackson Park Rd, Portland OR 97201 (E-mail: sukumarm@ohsu.edu).

J Thorac Cardiovasc Surg 2005;130:230-31

$0022-5223 / \$ 30.00$

Copyright $\odot 2005$ by The American Association for Thoracic Surgery

doi:10.1016/j.jtcvs.2004.11.054

ward on postoperative day 1 and discharged on postoperative day 3 after an uneventful course. Her symptoms, including nausea, lightheadedness, and presyncope, have not recurred to date, and she is doing well.

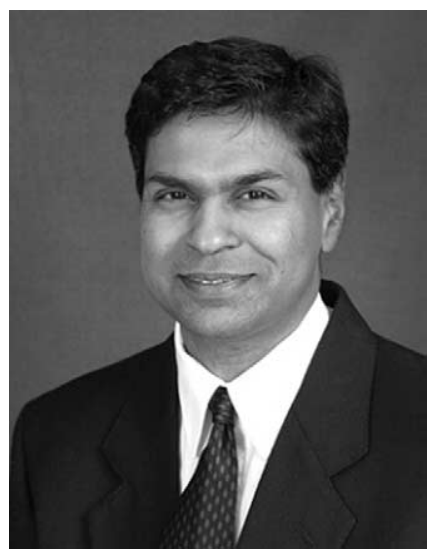

Dr Sukumar

\section{Discussion}

Cases with enlargement of the azygos vein can generally be divided into 3 categories. First are a few reported cases of traumatic disruption of the azygos vein, resulting in pseudoaneurysm formation. Second are cases of fusiform enlargement of the vein as a result of pressure or volume load, such as in portal hypertension or inferior vena caval occlusion, either congenital (azygos vein continuation syndrome) or as a result of neoplasm. In situations of volume or pressure overload, resection is contraindicated. Finally, approximately 30 cases have been reported that are likely attributable to failure of regression of the right supracardinal vein. ${ }^{2}$ Although some authors have reported observation of these lesions, resection might be necessary if the lesion is symptomatic, progressively enlarging, or requires diagnosis. This has been done previously through a right thoracotomy. ${ }^{3}$

Past reports have clearly described the safety of resection in cases that are not related to increased azygos flow or portal hypertension. Some authors have stated that thoracoscopic resection would be ideal, ${ }^{4,5}$ and there are 2 reported cases of thoraco-

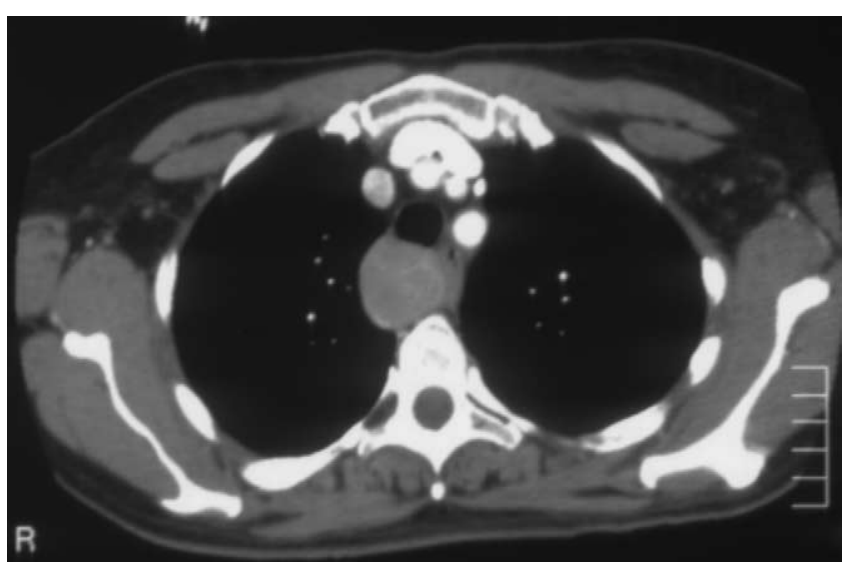

Figure 1. Computed tomographic scan of the chest demonstrating a posterior mediastinal mass with mild contrast enhancement. 


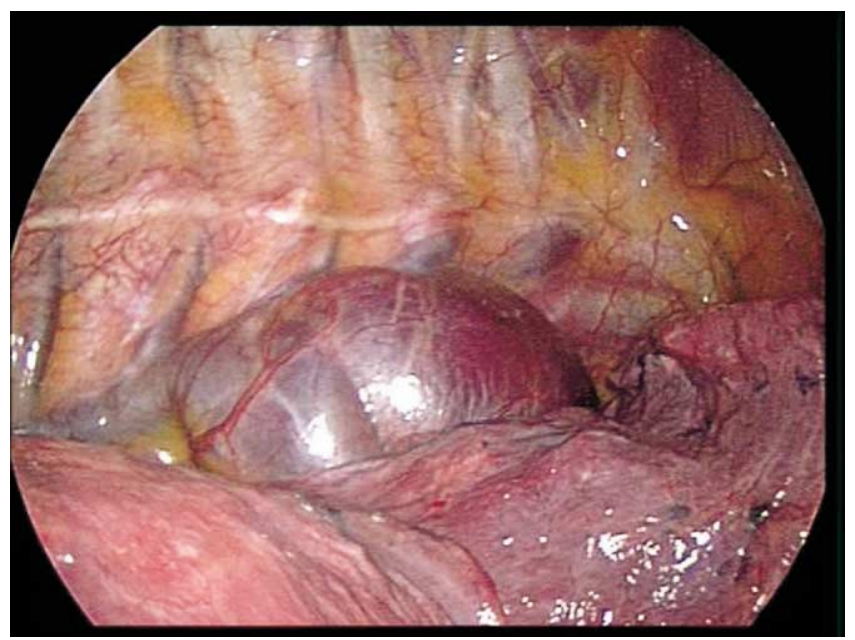

Figure 2. Azygos vein aneurysm before dissection.

scopic visualization of an aneurysm. Neither case proceeded with thoracoscopic resection. In one case injury to the vessel wall required conversion to thoracotomy, ${ }^{4}$ and in the other the authors declined to resect the aneurysm in an elderly woman. ${ }^{5}$ After an extensive literature review, we believe that this is the first reported case of successful thoracoscopic resection.
A second important issue is whether to resect such an aneurysm. In many cases the diagnosis might be difficult to make preoperatively. Even in situations in which it is made preoperatively, the natural history of such an aneurysm is unclear. In one case report the aneurysm was watched and enlarged, ultimately requiring resection. ${ }^{3}$ In symptomatic patients such as ours or in which the diagnosis is unclear, we believe resection is indicated. It might be reasonable to observe an asymptomatic azygos vein aneurysm because rupture has not been reported. Essentially, the decision to resect must be made on an individual basis.

In conclusion, although azygos vein aneurysms are a rare clinical problem, their consideration in the diagnosis of a posterior mediastinal mass is important. If resection is necessary, we would advocate a thoracoscopic approach because it is feasible and appropriate.

\section{References}

1. Gomez MA, Delhommais A, Presicci PF, Besson M, Roger R, Alison D. Partial thrombosis of an idiopathic azygos vein aneurysm. $\mathrm{Br} J$ Radiol. 2004;77:342-3

2. Gallego M, Mirapeix RM, Castaner E, Domingo C, Mata JM, Marin A. Idiopathic azygos vein aneurysm: a rare cause of mediastinal mass. Thorax. 1999;54:653-5.

3. Podbielski FJ, Sam AD 2nd, Halldorsson AO, Iasha-Sznajder J, Vigneswaran WT. Giant azygos vein varix. Ann Thorac Surg. 1997;63:1167-9.

4. Bobbio A, Miranda J, Gossot D, Perniceni T, Grunenwald D. Azygos vein aneurysm as a diagnostic pitfall. The role of thoracoscopy. Surg Endosc. 2001;15:1049-50.

5. Hatton PD, Manjoney DL. Giant azygos vein varix. Ann Thorac Surg. 1997;64:1527-8.

\section{Availability of Journal back issues}

As a service to our subscribers, copies of back issues of The Journal of Thoracic and Cardiovascular Surgery for the preceding 5 years are maintained and are available for purchase from Elsevier Inc. until inventory is depleted. Please write to Elsevier Inc., Subscription Customer Service, 6277 Sea Harbor Dr, Orlando, FL 32877, or call $800-654-2452$ or $407-345-4000$ for information on availability of particular issues and prices. 